\title{
Do Different Groups Have Different Epistemic Intuitions? A Reply to Jennifer Nagel ${ }^{1}$
}

\author{
Stephen Stich \\ Dept. of Philosophy \& Center for Cognitive Science \\ Rutgers University
}

\section{Introduction}

Intuitions play an important role in contemporary epistemology. Over the last decade, however, experimental philosophers have published a number of studies suggesting that epistemic intuitions may vary in ways that challenge the widespread reliance on intuitions in epistemology. In a recent paper, Jennifer Nagel (2011) offers a pair of arguments aimed at showing that epistemic intuitions do not, in fact, vary in problematic ways. One of these arguments relies on a number of claims defended by appeal to the psychological literature on intuitive judgment and on mental state attribution (a.k.a. "theory of mind", "mindreading" and "folk psychology"). I'll call this the theoretical argument. The other argument relies on recent experimental work carried out by Nagel and her collaborators. I'll call this the experimental argument. It is my contention that in setting out her theoretical argument, Nagel offers an account of the relevant scientific literature that is, in crucial respects, flawed and misleading. My main goal in this paper is to rectify these errors and to make it clear that, once this is done, Nagel's theoretical argument collapses. I am also inclined to think that Nagel's experimental argument is far from convincing. Although I will have a bit to say about that in the final section of this paper, a detailed discussion would be inappropriate at this time, since, although she has invoked her results in several publications (Nagel, $2011 \&$ forthcoming), the study itself has not been published (or posted on the internet) and many crucial details required for a careful assessment are not included in the papers in which Nagel sketches her results.

Here's how I propose to proceed. In section 1, I will distinguish two quite different epistemological projects in which intuitions are taken to be evidence, and explain why it would be problematic for one of these projects if epistemic intuitions vary in different groups. Section 2 is a brief discussion of some of the problems that arise in interpreting the findings in experimental philosophy. The next three sections are devoted to setting out and assessing Nagel's theoretical argument. In Section 3, I explain how she characterizes intuition. In section 4, I sketch Nagel's account of the psychological mechanisms underlying intuition, which is based on the work of Asher Koriat. In Section 5, the longest and most important section of the paper, I focus on two crucial claims in Nagel's theoretical argument:

(i) intuitive knowledge and belief attributions are made by the mindreading capacity

\footnotetext{
${ }^{1}$ I am grateful to Wesley Buckwalter, Gilbert Harman, Daniel Kelly, Joshua Knobe, Edouard Machery, Shaun Nichols, Jonathan Weinberg and Tomasz Wysocki for their helpful feedback on earlier drafts of this paper, and to Clark Barrett for his valuable guidance on the cross-cultural mindreading literature.
} 
(ii) the mindreading capacity is cross-culturally universal.

I argue that when "cross-culturally universal" is understood in the way required by Nagel's argument, there is no interpretation of "mindreading capacity" on which both of these claims are supported by the mindreading literature. Nagel's claims about that literature are simply mistaken. In the final section, I will make a few brief observations about Nagel's strategy for dealing with experimental data that does not support her view - both other people's and her own.

\section{Why the Experimental Studies of Epistemic Intuition Pose a Challenge}

Why do some experimental studies of epistemic intuitions pose a challenge to the use of intuitions in epistemology? Here is Nagel's answer.

Contemporary epistemologists employ various methods in the course of articulating and defending their theories. A method that has attracted particular scrutiny in recent years involves the production of intuitive responses to particular cases: epistemologists describe a person making some judgment and then invite their audience to check this judgment's epistemic status for themselves. "Does $\mathrm{S}$ know that P?" - A well constructed case can elicit a powerful intuitive verdict....

Experimental philosophers have suggested that close attention to the mechanics of intuitive judgment should make us uneasy about the method: intuitions may exhibit worrisome instability either within an individual (Swain, Alexander \& Weinberg, 2008, 335), or between groups of individuals, perhaps along such epistemically scary fault lines as ethnicity (Weinberg, Nichols \& Stich, 2001) or gender (Buckwalter \& Stich, 2011). If a certain intuitive response to some Gettier case works for the majority of epistemologists, we have no guarantee that it will work for others outside our professional club, and no good reason - the experimentalists argue - to suppose that it supplies genuine evidence about the nature of knowledge. ${ }^{2}$

This is, I think, a good first pass at setting out the challenge posed by the sorts of findings discussed in the papers that Nagel cites. But there are a number of additional points that need to be made to facilitate the discussion to follow.

The first is an observation about what epistemologists take intuitions to be evidence for Nagel's focus, in the last sentence of the passage I've quoted, and throughout her paper, is on the use of intuitions as "evidence about the nature of knowledge". She is surely right that many epistemologists take themselves to be in the business of giving an account of the nature of knowledge, and that they use intuitions like those that she describes as an important source of evidence for (or against) such accounts. However, not all epistemologists accept this characterization of their project. Some epistemologists instead take themselves to be in the business of conceptual analysis. Their goal is to characterize the concept of knowledge, and other important epistemological concepts, like justification, warrant or rationality, and they use

\footnotetext{
${ }^{2}$ Nagel (2011), manuscript page 1. Additional references to this paper will be given in parentheses in the text.
} 
intuitions as evidence about the nature of these concepts. This view, which has been defended most systematically by Alvin Goldman (2007, 2010), is sometimes taken to be incompatible with the view that epistemologists use intuitions as evidence about the nature of knowledge. I am inclined to think that, when couched as a disagreement about what epistemologists are really doing, the debate is rather silly. Most epistemologists are pretty smart and pretty reflective people. So if one epistemologist says she is interested in characterizing the nature of knowledge and another says he is interested in characterizing the concept of knowledge, I see no reason not to take both of them at their word. There are, of course, complex and contentious connections between the two projects. And there are substantive - and importantly different - arguments about the legitimacy of using intuitions as evidence in each project. But I raise these themes only to make clear that, in this paper, I propose to set them aside. Nagel's focus is on the epistemologist whose goal is to determine the nature of knowledge, and on the use of intuitions as evidence in that project. In what follows, that will be my focus as well.

Why would it be problematic for such an epistemologist if groups of people, divided perhaps by "epistemically scary fault lines" like ethnicity or gender, have different intuitive responses when presented with Gettier cases, or other cases that have loomed large in the recent epistemological literature? Though a detailed answer would be a long and controversial story (Weinberg, 2007), the basic point is clear enough. When a philosopher uses intuitions as evidence about the nature of knowledge, she typically assumes that the content of the intuition is true. If people have the intuition that the protagonist in a Gettier case does not know that $\mathrm{P}$, the epistemologist takes this to be evidence that the protagonist doesn't know that P. So an account of the nature of knowledge - like the traditional justified true belief account - that entails the protagonist does know that $\mathrm{P}$ is challenged, while an account that entails the protagonist does not know that $\mathrm{P}$ is supported, or at least unthreatened. But if one group has the intuition that the protagonist does know that $\mathrm{P}$ and the other group has the intuition that the protagonist does not know that $\mathrm{P}$, then they can't both be right. And if these group differences are found in a significant number of cases of the sort that epistemologists appeal to in debating the virtues of competing accounts of knowledge, then the assumption that the content of people's intuitions in philosophically important cases are usually true is threatened, and the practice of using these intuitions as evidence about the nature of knowledge is undermined. Though much more could be said about all this, ${ }^{3}$ there is no need to do that here, since Nagel's theoretical and experimental arguments do not turn on the fine points of how the experimentalists' challenge is spelled out. Rather, her claim is that the alleged intuitive disagreements - particularly those along "epistemically scary fault lines" - have been seriously exaggerated. According to Nagel, "neither ethnicity nor gender has a significant impact on knowledge ascription in general, nor on epistemologically interesting cases in particular."(3)

\section{Some Problems in Interpreting Findings in Experimental Philosophy}

Work in experimental philosophy of the sort that Nagel has in mind typically uses surveys in which participants read a brief vignette and are then asked about what one of the protagonists in the vignette knows. If one participant indicates that the protagonist knows that $\mathrm{P}$ and another indicates that the protagonist does not know that $\mathrm{P}$, this is taken as evidence that the

\footnotetext{
${ }^{3}$ See Nado (2011) for an insightful discussion.
} 
participants have different epistemic intuitions. However, as a number of authors have noted, there are many reasons why this inference might be problematic. Early on in the debate over the implications of experimental philosophy, Sosa (2009) noted that different people - and more importantly different groups of people - may interpret a vignette in different ways because they make different assumptions about the characters in the vignettes, about the situation described, or about what they are being asked to do. In other cases, a participant or a group of participants may simply not be paying attention. In still other cases, people may misreport their intuitions because prevailing norms make the content of the intuition problematic. (Robbins \& Rumsey, 2008) In experimental philosophy, as elsewhere in the social sciences, it is all but impossible to design an experiment that rules out all the imaginable confounds. However, additional experiments with different designs, different controls, different instructions or differently worded vignettes can help to address most of these concerns. As is typically the case both in the social sciences and in science more generally, progress is made incrementally as plausible alternative explanations of experimental findings are themselves tested empirically.

A problem of a rather different sort arises when a crucial term in a question put to participants has different meanings (or expresses different concepts), or has different extensions for different groups of participants. This is almost always a salient concern when surveys are conducted in different languages. But it can also arise when all the participants are speakers of the same language (Sosa, 2009, 2010). The issue can be particularly vexing because there is no consensus, either among philosophers or among linguists, about what constitutes a difference in meaning, or about how concepts are to be individuated. For Frank Jackson, for example, if two people disagree about standard Gettier cases, and neither of them is confused about the details of the vignette, then they simply mean different things by 'knowledge' (or 'know') - they have different concepts of knowledge. (Jackson, 1998, p. 32) For Fodor (1998), on the other hand, at least in some of his moods, there is hardly any disagreement of this sort that would justify the conclusion that the participants mean different things by 'knowledge' or that they are expressing different concepts. I am inclined to think that the challenge posed by experimental philosophy can sidestep this disagreement about meaning (and similar disagreements about reference). But that's a topic for another occasion. For current purposes, the point to keep in mind is that there is no agreement on what counts as a difference in meaning or in concepts, and that when the issue is important, as will be in Section 5, we may need to make a "judgment call". 4

\section{Nagel's Theoretical Argument: Part I - An Account of Intuition}

Nagel's theoretical argument, which is the main focus of this paper, has several parts. In the first she endorses an account, suggested by Mercier and Sperber, of what an intuition (or an intuitive judgment) is, and argues that the sorts of epistemic intuitions that are typically invoked in philosophical arguments are intuitive in this sense. "Mercier and Sperber describe intuitive judgments as generated by 'processes that take place inside individuals without being controlled by them' (Mercier \& Sperber, 2009, 153). The spontaneous inferences produced by these processes modify or update what we believe 'without the individual's attending to what justifies this modification' (ibid.)."(4) "In intuitive judgment," Nagel tells us, "we are 'conscious only of the results of the computation, not the process.' (Sloman, 1996).”(4) Intuitive judgments are

\footnotetext{
${ }^{4}$ For a related and more detailed discussion, see Machery (2008), §2.
} 
contrasted with reflective judgments that we produce through deliberate and explicit reasoning. In reflective judgment, "we engage in explicit reasoning and devote personal-level attention to the grounds of the conclusions we reach."(4) Nagel's examples of intuitive judgment include "automatically and effortlessly recognizing the face of a friend" and "producing an answer when one is asked to sum $2+2$."(5) Her examples of reflective judgment include explicit syllogistic reasoning and long division. Importantly, Nagel recognizes that new intuitions can be acquired: "Judgments naturally become intuitive when made very frequently."(7) Thus, for example, "it should be possible to have theory-driven epistemic intuitions ... after becoming very wellrehearsed in applying the verdicts of some particular analysis of knowledge."(8)

To show that our judgments about whether a particular case is an instance of knowledge are usually intuitive, Nagel marshals a number of observations. "Most notably, we do not need to possess or apply any explicit theory of knowledge in order to gain the sense that the protagonist of some scenario has or lacks knowledge. We are not fully conscious of the grounds of our judgment in the way that we would be if we were making a reflective categorization on the basis of an explicit theory." (7)

There is a substantial philosophical literature debating how best to characterize the sorts of intuitions that do or should play a role in philosophical argument (Nado, 2011; Alexander, forthcoming, ch. 1). And while many authors would insist that not all of the epistemic judgments that Nagel would count as intuitive should be counted as philosophically kosher intuitions, I think most authors would agree that everything they would count as a proper philosophical intuition would also count as an intuition on Nagel's account. Certainly, I would have no qualms about this. So for the remainder of this paper I will assume, without further discussion, that the sorts of judgments about cases that are typically invoked as evidence in epistemological arguments are intuitive, in the sense that Mercier, Sperber and Nagel have sketched.

\section{Nagel's Theoretical Argument: Part II - The Psychological Mechanism Underlying Intuition}

In the next part of her argument, Nagel offers an account of the psychological mechanism underlying philosophical intuitions. The account, based on the Self-Consistency Model developed by Asher Koriat (1975, 1976, 1995, 2008, 2011), also offers an explanation for the putative fact that when philosophical intuitions "feel clear and strong" they are "likely to be felt by others.'(9) Koriat's model was designed to explain a cluster of intriguing findings about twoalternative forced-choice tasks, like guessing which of two squiggly line segments is longer, or matching pairs of antonyms in an unfamiliar language to their English translations. In this latter task, participants might be asked to make their best guess about which of the pair [tuun, luk] means "deep" and which means "shallow". After responding, participants are asked to rate their confidence, which Koriat describes as "the unexplainable feeling that you may be correct."(10) Koriat found that "accuracy rose monotonically with confidence rating."(10) In subsequent work, Koriat showed that, in a range of tasks, confidence predicts "consensuality" - the likelihood that others will make the same response - and it also predicts the likelihood that a given participant will repeat that response when presented with the same two-alternative forcedchoice task on a later occasion. 
Here is Nagel's account of the Self-Consistency Model (SCM), which Koriat and colleagues developed to explain these findings.

According to the SCM, subjective confidence is a byproduct of the process of intuitive judgment. The parallel processing that underpins intuitive and perceptual judgment draws on a vast range of information potentially relevant to the problem at hand. We do not consult the entire range on any given occasion. In generating an answer to a two-alternative forced-choice question, a sample of representations is drawn from the pool in order to produce a response, where each representation is some consideration in favor of one or the other answer. Sampling continues until either a critical number of representations favoring one side has been amassed, or until a preset number of samples has been drawn. One's response is determined by the direction of the sample's majority, and one's confidence in that response is determined by the size of the sample's majority, the internal consistency of the sample in one direction or the other.... On this model, neither one's answer nor one's confidence level will directly measure the extent to which the underlying pool of information supports an answer to the question: it is possible to draw an unrepresentative sample from a pool which overall strongly favors $\mathrm{P}$, and respond that not-P. However, when we have done such a thing, the odds are overwhelming that our sample will speak only weakly in favor of the unrepresentative choice. If the pool strongly favors $\mathrm{P}$, we are likely to draw a very consistent sample from it, and respond with higher confidence for P. If confidence is determined by the consistency of one's sample, then confidence will predict the likelihood of one's making the same choice on subsequent occasions. Furthermore, if others are drawing from a similar pool of representations, individual confidence will predict consensuality as well: a choice very heavily favored by one's own sample is likely to be favored by others also. (11-12, emphasis added)

According to Nagel, "the SCM is a general model of intuitive judgment for two-alternative forced-choice questions," and since the question asked at the end of a typical epistemological thought experiment - "Does S know that P?" - is a two alternative forced-choice question, Nagel speculates that "the SCM model applies to intuitive judgments in epistemology as it applies to other types of intuitive judgments."(13) She goes on to note that the applicability of the SCM to epistemic intuitions would help to explain the dialectical success of appeals to intuition in philosophy, since if a philosopher has a strong intuition about a particular case, it is likely that other philosophers will share the intuition, provided that the underlying pool of representations that the two philosophers have available is similar.

Nagel does not claim that there is any direct evidence in favor of the speculation that the Self-Consistency Model accounts for epistemological intuitions. It is, she acknowledges, "conceivable that intuitive judgments in epistemology are managed in some quite different way." But she believes that "we have no positive reason to think so." I am far from convinced that the SCM can be extended to intuitive judgments in epistemology or to intuitive judgments in other areas of philosophy. When it comes to philosophical intuitions, I think it is wise to be a splitter 
rather than a lumper. My splitter inclinations are encouraged by a growing body of work suggesting that philosophical intuition is a heterogeneous category, and that philosophical intuitions are subserved by a variety of quite different psychological and neurological mechanisms. (Cushman et al., 2010; Nado, 2011; Sinnott-Armstrong \& Wheatley, under review) But this is not the place to pursue that issue. My main criticisms of Nagel's theoretical argument lie elsewhere. So for current purposes, I propose to put this concern to one side and to grant, though only for argument's sake, that Nagel's speculation is correct and the SCM offers a plausible account of the psychological mechanism underlying the production of epistemic intuitions.

Note, however, that by itself the SCM tells us nothing at all about how likely it is that two people, or two groups of people, will have different epistemic intuitions. Nor does it tell us how likely it is that two people, or two groups of people, will have different strong intuitions i.e. intuitions about which they are highly confident. As I emphasized with italics in the long quote above, the Self-Consistency Model only predicts that strong intuitions are likely to be shared if the people producing the intuitions base them on similar pools of relevant representations. If culture or gender or philosophical training has a significant impact on the pool of representations that the SCM can draw on when generating intuitions in a given area, then it might well be the case that the intuitions produced in the members of one group are different from the intuitions produced in members of another, and that people in each group are very confident about their intuitions.

The cross-cultural literature on perceptual illusions offers a particularly striking example of this phenomenon. Nagel spends several pages discussing perceptual illusions because she thinks that the SCM can explain the fact that "our intuitive responses to classic illusions such as the Müller-Lyer" are "robustly and consensually wrong" (13, emphasis added). Though claims about "our" judgments and intuitions, and about what "we" think or do, occur with some frequency in Nagel's paper, she does not tell us who "we" and "our" includes. But apparently it does not include the San foragers of the Kalahari, since a classic study of 16 cultures by Segall et al. (1966) showed that San adults are unaffected by the Müller-Lyer illusion. For them, as Henrich et al. (2010) note, it is not an illusion at all. American participants, by contrast, were at the extreme opposite end of the distribution. Both children and adults were significantly more susceptible to the illusion than participants from all of the other cultural groups included in the study. ${ }^{5}$ If, for argument's sake, we assume that the Self-Consistency Model explains people's intuitive judgments about the Müller-Lyer (and the other standard illusions that Segall and colleagues studied), then the most natural explanation of the Segall et al. findings is that the "pool of relevant representations" is different in different cultures. ${ }^{6}$

\footnotetext{
${ }^{5}$ For a striking overview of the Segall et al. data, see Henrich et al. (2010), Figure 2.

${ }^{6}$ Though Nagel launches her discussion of perceptual illusions by mentioning the Müller-Lyer, she focuses on "some fresh cases" used by Koriat. None of these were used in the Segall et al. study, and Koriat reports no crosscultural data. (It appears that all of his participants were students at the Hebrew University and the University of Haifa.) So we do not know whether these illusions would vary from one culture to another. But what the Segall et al. evidence indicates is that some perceptual illusions, including the Müller-Lyer, vary dramatically.
} 


\section{Nagel's Theoretical Argument: Part III - Knowledge and Belief Attributions are Generated by the Mindreading System, and Mindreading is Cross-culturally Universal}

In the next step of Nagel's theoretical argument, she makes a pair of crucial claims. The first is that intuitive attributions of knowledge and belief are made by the "mindreading capacity." The second is that this capacity is cross-culturally universal. If these claims are true, then we should expect epistemic intuitions to be universal as well. So it would be reasonable to conclude that studies in which participants from different groups report different epistemic judgments are beset by problems like those discussed in the first paragraph of section 2, and thus that the judgments reported by one or the other group (or both) are not an accurate reflection of their epistemic intuitions. Unfortunately, Nagel's discussion of these two crucial claims goes by rather quickly, taking up only a bit over two pages. ${ }^{7}$ It is my contention that what Nagel says in these two pages is far from convincing. More specifically, I maintain that each of her two crucial claims can be interpreted in a variety of ways. And when we focus on the interpretations required to support the conclusion that studies reporting group differences in epistemic intuition are suspect, Nagel does not come close to making a convincing case.

I'll start with the claim that intuitive knowledge attributions are generated by our mindreading capacity. Here is what Nagel says:

How do we attribute knowledge or mere belief to the protagonist of an epistemological scenario? The default answer to this question is: the same way we generally attribute knowledge or belief to anyone else. Unless there is a special reason to think that knowledge attributions work quite differently when we are reading philosophy papers ... we should expect to find that epistemic case intuitions are generated by the natural capacity responsible for our everyday attributions of states of knowledge, belief, and desire. This capacity has been given various labels, including 'folk psychology', 'mindreading' and 'theory of mind'. I will follow Ian Apperly and others in calling this capacity 'mindreading', but intend to remain as neutral as possible about the current debates within psychology about the exact nature of this capacity.

Although a great deal of work in mindreading has focused on natural illusions of mental state attribution, particularly in children whose capacities are still immature, it is generally agreed that adult capacities for mental state ascription provide fairly reliable tracking of what others think and know. (16)

It is uncontroversial in psychology to see intuitive attributions of knowledge as falling under the scope of our mindreading capacity, so anyone who generally trusts that latter capacity has reason to trust intuitive knowledge ascriptions. (18)

Her case for the claim that the mindreading capacity is a cross-cultural universal is comparably brief:

\footnotetext{
${ }^{7} \mathrm{Pp} .16-18$ in the manuscript available on-line.
} 
Mindreading capacities are generally thought to be cross-culturally universal, not least because of developmental similarities in mindreading in radically different cultures (Wellman, Cross \& Watson, 2001). Natural illusions within the mindreading capacity are also cross-culturally shared. [fn: In particular, we share a bias known as hindsight or more broadly epistemic egocentrism, a bias which distorts our mindreading of those who occupy a more naïve perspective, including our own more naïve past selves.] Furthermore, mindreading capacities are thought to exhibit no gender differences in typical adults; extensive research on mental state ascription has shown only a mild advantage for girls before the age of four, perhaps simply as a function of girls' earlier linguistic development, and no discernable gender differences in the non-clinical population beyond that point (Charman, Ruffman \& Clements, 2002). For those who suspect that there is cross-cultural variation in Gettier case recognition (following Weinberg et al. 2001), and for those who suspect that Gettier intuitions may not be felt equally by men and women (following Buckwalter \& Stich, 2011), it may seem unlikely that epistemologists' responses to Gettier cases would be produced by our common mindreading capacity.(17)

A first crucial question of interpretation focuses on Nagel's claim that "mindreading capacities are generally thought to be cross-culturally universal." There are two importantly different ways to understand this claim. To see the distinction, consider the language capacity or the perceptual capacity to judge the relative length of straight line segments. There is one sense in which both of these capacities are uncontroversially cross-culturally universal. People in all human cultures use language, and people in all human cultures can judge the relative length of line segments. But, of course, there is also a sense in which there are important cross-cultural differences in both the language capacity and the capacity to judge the relative lengths of line segments - and these differences reflect themselves in people's intuitions. My intuitions about which sentences are grammatical are somewhat different from the intuitions of a speaker of AAVE (African American Vernacular English) and radically different from those of a monolingual Tagalog speaker. More surprisingly, my intuitions about the relative lengths of Müller-Lyer line segments are significantly different from the intuitions of a Kalahari San bushman. Now let's consider mindreading. It is widely (though not universally ${ }^{8}$ ) agreed that people in all cultures have the capacity to attribute mental states to one another. So the mindreading capacity is generally thought to be cross-culturally universal in the way that the language capacity is generally thought to be cross-culturally universal. But, of course, this is not what Nagel needs for her argument. In order to make the case that epistemic intuitions are unlikely to vary from one group to another, it must be the case that the mindreading capacity is cross-culturally universal in the way that the language capacity and the capacity to judge the relative length of line segments are not. It is not enough that people in all cultures engage in mindreading, they have to do it in the same way. To keep track of this important distinction, I'll use the label "universal in the strong sense" for traits that are universal in the way required for Nagel's argument - the way in which the language capacity is not universal.

Is it really the case that the mindreading capacity is generally thought to be crossculturally universal in the strong sense required for Nagel's argument? Here, again, we confront

\footnotetext{
${ }^{8}$ For an overview of some dissenting views, see Robbins \& Rumsey (2008).
} 
a problem of interpretation. When Nagel talks about the "mindreading capacity" or the "mindreading capacities," which "natural capacity" or capacities does she have in mind? The question is a pressing one since, as Astington \& Baird (2005) point out, the term "theory of mind" (which, for Nagel, is an alternative term for "mindreading") has been used to refer to a wide range of abilities. The list they offer in a useful table labeled "The Multifacited Nature of Theory of Mind" contains 15 entries including:

- false belief understanding

- meta-representation

- belief-desire reasoning

- mental state attribution

- perspective-taking

- social intelligence

- social intuition

- social perception

- person perception

- intersubjectivity $^{9}$

I'll use the term "the mindreading capacity broadly construed" for any account of the scope of mindreading that includes most or all of these. If, when Nagel talks about the mindreading capacity, she means the mindreading capacity broadly construed, it would be hard to quarrel with the claim that epistemic intuitions are generated by this capacity. But, unfortunately for Nagel, on this reading of "mindreading capacities", it is simply not true that "mindreading capacities are generally thought to be cross-culturally universal."(17) Quite the opposite is the case. It would be easy to generate a long list of publications arguing that mindreading broadly construed exhibits enormous cross cultural variability. ${ }^{10}$ Indeed, if "mindreading capacities" include most of the items on Astington \& Baird's list, it is hard to believe that there is any reputable researcher who thinks that it is a cross-cultural universal in the strong sense required by Nagel's argument.

One of the items on Astington \& Baird's list is false belief understanding. And in Nagel's very brief defense of the claim that mindreading capacities are generally thought to be cross-culturally universal, two of the three papers she cites are focused exclusively on the emergence of this aspect of mindreading as children mature. So perhaps when Nagel talks about the mindreading capacity what she has in mind is the capacity to understand and attribute false beliefs, and whatever additional capacities come along with this. Here, though the literature does not speak with one voice, there does indeed appear to be an emerging consensus that the capacity to pass the false belief task is a human universal and that it emerges at about the same time in quite different cultures. One of the most persuasive and widely cited recent studies contributing to this consensus is Callaghan et al. (2005), which used closely matched culturally appropriate

\footnotetext{
${ }^{9}$ Astington \& Baird (2005), p. 5.

${ }^{10}$ Bauman \& Skitka (2006); Duranti (2008); Gauvain (1998); Harris (1990); Heine (2008), chs. 5 \& 8; Lillard (1997, 1998, 1999); Nelson (2005); Paul (1995); Robins \& Rumsey (2008); Rosen (1995); Vinden (1999). See also the passages quoted later in this section from Callaghan et al., Scholl \& Leslie, Wellman and Apperly.
} 
versions of the false belief task in five cultures: Canada, India, Peru, Samoa and Thailand. The authors report that "the fundamental shift in understanding the impact of false belief on behavior appears to be a universal milestone of development that occurs between 3 to 5 years of age...." and they conclude that this "undermines the claim that particular cultural views, such as a Western concept of mind, profoundly influence this very basic aspect of early mental-state reasoning, and strengthens a claim of universality." (Callaghan et al., 2005, 381) But, of course, the mere capacity to pass the false belief task does not by itself enable a person to attribute knowledge in the way (or ways) that adults do; indeed it doesn't enable a person to attribute knowledge at all. But perhaps Nagel thinks that if kids in all cultures acquire the capacity to pass the false belief task at roughly the same time, this lends support to the hypothesis that there will be lots of cross cultural universals in other mindreading capacities, including those that emerge later and that enable adults to make judgments about Gettier cases. Callaghan et al. disagree.

Synchrony in the age of onset of mental-state reasoning does not preclude diversity in outcome. Our findings support the view of Avis \& Harris (1991), who suggested that adults and children may have access to a universal understanding of belief-desire psychology even though they come to elaborate this understanding differently, depending on their culture's practices.... The present study establishes the existence of a common starting point for the very fundamental understanding that actions are based on representations of reality. We agree with other researchers (Avis \& Harris, 1991; Harris, 1990) who have suggested that reasoning on the basis of inferred belief systems is merely the beginning foundation of psychological understanding that is later supplemented with more complex forms of mental-state reasoning and with culturally specific principles of the causes of human action (Lillard, 1998). The sophisticated theories of mind held by adults in the cultures studied by these researchers, as well as in other cultures, are rich in their diversity, and the refinement of early fundamental understanding to later sophisticated nuance is undoubtedly the work of cultural influence. (383)

So for Callaghan et al. what is universal is just a common starting point for mindreading, a beginning foundation that is later supplemented with culturally specific principles. Cross culturally, adult theories of mind are rich in their diversity.

It is important to note that, despite differences in emphasis, this picture is widely shared among researchers with very different theoretical perspectives. Consider, for example, the following passage from Alan Leslie, who is perhaps the most trenchant defender of nativism in mindreading.

As the modular account thus predicts, the acquisition of ToM [theory of mind] is largely uniform across both individuals and cultures. The essential character of ToM a person develops does not seem to depend on the character of their environment at all. [Fn: For our purposes here, the 'essential character' of ToM refers to three basic concepts, BELIEF, PRETENCE, and DESIRE.] It is at least plausible, prima facie, that we all have the same basic ToM! .... 
A few authors have recently alleged the existence of cross-cultural differences in ToM (e.g. Lillard, 1997, 1998), but these allegations turn out not to be relevant to the modularity account. The cross-cultural differences catalogued by Lillard (1998) explicitly include differences in religious beliefs, and beliefs in phenomena such as witchcraft, magic and karma. As such, her view of crosscultural differences pertains only to the inessential flurescences of mature ToM competence, rather than to its essential character in early acquisition... The modular account of the acquisition of ToM explains the origin of the basic metarepresentational concepts (like BELIEF, PRETENCE and DESIRE), and not necessarily how these concepts may be employed by different extramodular cognitive processes in mature individuals.... Wellman (1989) offers a similar analysis of this recent work:

theory of mind research is pitched, fundamentally, at attempting to capture deeper core construals... Such an attempt may be mistaken, but it would not be derailed by the evidence Lillard (1998) amassed... Resulting folk psychologies could be quite different from one another worldwide; each could be quite removed and advanced beyond, although grounded in, the initial framework assumptions of young children... [V]ariability in adult folk psychologies, of the sort that Lillard has catalogued, may say little about the existence, nature of development of core folk psychological conceptions. (Scholl \& Leslie, 1999, 136-138, emphasis in the original)

So for Scholl and Leslie, what is cross-culturally universal are basic metarepresentational concepts like BELIEF, PRETENCE and DESIRE. How these concepts are employed by extramodular cognitive processes in mature adults may vary widely from culture to culture. While the rhetoric is rather different, the picture that Scholl and Leslie - and Wellman - are endorsing is much the same as the view in Callaghan et al.: an early emerging core of "metarepresentational concepts" or "initial framework assumptions" that can develop into adult folk psychologies that are "quite different from one another worldwide."

Yet another author who adopts a similar picture is Ian Apperly, from whom, it will be recalled, Nagel adopted the term "mindreading," and to whose work she refers frequently in her paper. In his important recent book, Mindreaders (2011), Apperly sets out a "two-systems" account of the processes underlying the attribution of what he calls "epistemic mental states" which he characterizes as "mental states such as perceptions, knowledge and belief" (Apperly, 2011, p. 4; subsequent references to Apperly, 2011, will be cited as A followed by the page number). On Apperly's account, there are two sorts of mindreading processes: low-level and high-level. Low level mindreading is subserved by two sorts of modules: original modules, which "we are pre-destined to develop"11 (A121) and modules that result from a process of "downward modularization" which "extracts regularly-occurring aspects of [a previously learned mindreading] ability into a schema of input-output relations that can operate efficiently but inflexibly." "12 (A123) High level mindreading involves a wide range of non-modular processes,

\footnotetext{
${ }^{11}$ For reasons that need not concern us, he avoids the term "innate".

${ }^{12}$ This is similar to the process that Nagel mentions, in which "judgments naturally become intuitive when made very frequently," (7, quoted in $\S 4)$, though she does not cite Apperly in this context.
} 
and "a large amount of the heavy lifting is performed by structured knowledge contained in scripts, narratives and social norms. Many of these are essentially social in origin, and variation in such knowledge over age or across individuals is likely to make a significant difference not only to general social skills but specifically to the accuracy of mindreading.,"13(A132) Low-level mindreading processes that are subserved by original modules are likely to be cross-culturally universal, ${ }^{14}$ while processes that can be traced to downward modularization may not be. More importantly, high level processes will often vary from culture to culture, since they are dependent on "scripts, narratives and social norms" many of which are very culturally local indeed. So, in general terms, Apperly's account is consonant with those urged by Callaghan at al. and by Scholl \& Leslie and Wellman: there is a cross-cultural core of mindreading skills that emerge quite early in development, and a great deal of variation in those aspects of mindreading that go beyond the core.

There is, however, one respect in which Apperly's account of mindreading might be thought to be much more congenial to Nagel's theoretical argument than Leslie's account. For, while Leslie typically does not included KNOWLEDGE on his list of metarepresentational concepts supplied by the innate theory of mind module, Apperly maintains that some knowledge ascriptions are subserved by original modules. "Simple ascriptions of perception, knowledge and belief," he tells us, "are achieved via cognitively efficient 'low-level' processing modules, at least some of which develop early and may be shared by humans and non-human animals." (A109) The empirical work that leads him to this conclusion is fascinating and important. Apperly provides an excellent overview.

Hare et al.'s (2000) paradigm provides evidence that chimpanzees track what each other see and use this to determine their strategies for food competition. Hare, Call and Tomasello (2001) adapted this paradigm to test whether chimpanzees also track what one another has seen and thus, in a sense, what the other "knows". As shown in Figure [1], a subordinate subject and a dominant competitor faced one another across a space that now had two barriers, and thus two possible locations where food could be hidden out of view of the dominant competitor. In a baseline condition both animals saw food being hidden behind one barrier. In the test condition, only the subject saw the food being hidden. Thus, in both conditions, at the point when the subject chimpanzee had to decide whether to approach the food, the subject chimpanzee could see the food and the dominant competitor could not. What varied was whether or not the dominant chimpanzee had seen the food being hidden. The result showed that subject chimpanzees were more likely to approach and obtain the food when the competitor had not seen the food being hidden (and so did not "know" it was there), compared to the baseline where both animals saw....

\footnotetext{
${ }^{13}$ It is important to note that Apperly's distinction between high level and low level mindreading does not map on to the Mercier, Sperber and Nagel distinction between intuitive and reflective processes. Some of Apperly's examples of high level mindreading processes are reflective. But others, like "Level-2 perspective taking" tasks, are clearly intuitive.

${ }^{14}$ Though see the caveat in the last quote from Apperly in this section.
} 


\section{FIGURE 1 ABOUT HERE}

Of course, "seeing" or "knowing" are relations that obtain between an agent and an object or situation, whereas the results I have described so far could be explained by a simpler strategy of just tracking properties of the food object itself. If, as a result of being seen, the food was tagged in the animal's memory as "seen", or even in entirely non-mentalistic terms as "low quality" or "dangerous", then the animal would behave in the way observed without processing any enduring relation of the dominant chimpanzee "knowing" about the object. It is significant, then, that in a second experiment, Hare et al. (2001) found that subject chimpanzees' reluctance to approach food that had been seen by a dominant competitor was eliminated if the competitor who had seen the food being hidden was replaced by another dominant animal who had not seen. This would not be expected if the subject chimpanzee had encoded the fact that the food had been seen only as a fact about the food. Rather, their strategy involved tracking a relationship between the food and a particular dominant individual. (A48-9)

Apperly goes on to speculate that the original modules devoted to mindreading that we humans may share with some non-human animals can be fruitfully compared to the "core knowledge" systems for enumerating small sets of objects and estimating the magnitude of large sets of items that has been explored in the influential and widely known work of Carey, Spelke, Dehane and others (Feigenson, Dehane \& Spelke, 2004; Spelke \& Kinzler, 2007; see A43-4 \& A149-151). What makes the analogy important for present purposes is that, if these researchers are right, these core knowledge systems continue to operate in adults. So if the analogy is a good one, all normal human adults will share the still active original module responsible for the attribution of knowledge by chimpanzees in the Hare et al. (2001) study. Thus there is, indeed, a cross-culturally universal component of the mindreading system that is responsible for the attribution of knowledge and that remains active in adults.

There are, however, several quite different reasons why I don't think Nagel can take much comfort from this part of Apperly's account of mindreading. The first turns on the question of what, exactly, is attributed to the "target" of mindreading by the original module whose existence Apperly so deftly defends. As the reader will no doubt have noted, Apperly puts the words "know" and "knowing" in quotes throughout the long passage quoted above. In a footnote a few pages earlier, he explains why.

It seems felicitous to use the normal terms for these mental states of perception, knowledge and belief, but I place them in scare-quotes in recognition of the fact that - as discussed later - they almost certainly do not correspond fully with the adult human's understanding of these terms. (A56, fn 4)

In those later discussions (A52 \& A146-7), Apperly sets out a number of ways in which the chimpanzee's understanding of "knowledge" "falls qualitatively short of the conditions of the normative account."15 Perhaps the most important of these is that, on his reading of the

15 The normative account is Apperly's term for the understanding of mindreading concepts that prevails in philosophy. (A5) 
evidence, the chimpanzee's concept of "knowledge" is not a relation between an agent and a proposition; rather, he suggests, it is a "relationship between agents, objects and properties." (A147; see also Apperly \& Butterfill, 2009) As I noted at the end of section 2, there is little agreement on how to determine whether a pair of concept tokens have the same content or the same reference, and thus that the issue will often come down to a "judgment call". My call, and Apperly's, is that the concept of "knowledge" that the chimps are invoking in the Call et. al (2001) experiments has neither the same content nor the same reference as the concept (or concepts) that adult humans invoke in making everyday knowledge attributions or in making judgments about Gettier cases and other philosophically important cases. ${ }^{16}$

However, even if Apperly and I are wrong about this, Apperly's account clearly does not support the conclusion that Nagel needs, since it does not suggest that adults in different cultural groups will agree in their knowledge attributions. One reason for this is that, as Apperly notes, the analogy with number cognition actually points in the opposite direction.

To date there is no evidence concerning variability in low-level mindreading within typically developing individuals. To the degree that the architecture and processing characteristics of low-level mindreading processes are tightly constrained by genetic and environmental input, we might expect these processes to be ubiquitous in the typical population, and the level of individual variability in these processes to be low. However, the case of number cognition suggests that variability may exist, even in a cognitive processes that is universal in humans and shared with many non-human species. Recall that one of the two low-level processes for number cognition allows the efficient estimation of the magnitude of large sets of items. Although this process always yields an estimation, the accuracy of the estimation does vary across individuals and, moreover, this variability is related to performance on "high-level" symbolic number problems (Gilmore \& Inglis, 2009). It may well be that similar effects will be found for mindreading. (A164)

A second and more important reason is that, on Apperly's theory, when a conflict arises, high level mindreading processes, with their reliance on scripts, narratives and social norms, typically override the low-level system in the production of the sort of "explicit [i.e. verbally expressed] judgments required by standard mindreading tasks". (See the discussion on A153-5; the quote is on A153-4). Moreover, there is only a "relatively narrow range of problems that can

\footnotetext{
${ }^{16}$ As Apperly notes at a number of places, similar problems arise when ordinary mental state terms are used in interpreting studies of mindreading in children.

Thinking about children's mindreading has been dominated by the view that children's main problem is to acquire a certain set of concepts, such as "belief", "desire" and "knowledge"... But describing children's mindreading development as the acquisition of concepts explains less than we might have hoped about mindreading because it remains unclear what it means to be the owner of such concepts, how we tell who has them, and how anyone puts these concepts to use in everyday mindreading. Moreover, since it is typically assumed that mindreading concepts are acquired in early to mid childhood, this approach offers little insight into the mindreading abilities of older children or adults. (A156, emphasis added; see also A109)
} 
be processed successfully by a low-level processing module." The high level processes "yield an enormous extension to the limited mindreading that can be achieved with modular processes." (A155) While Apperly never mentions Gettier cases, True Temp cases or anything much like them, it is clear that these are far beyond the "narrow range of problems" that a low-level module could process. The bottom line, then, is that Apperly's account of mindreading lends no support at all to the claim that the output of the mindreading system - the judgments it generates - will be cross-culturally universal when those judgments are attributions of knowledge, or when they are the attribution of any other mental state.

This has been a long section. It's time to sum up. My goal, in this section, was to rebut two crucial claims in Nagel's theoretical argument:

(i) intuitive knowledge and belief attributions are made by the "mindreading capacity"

(ii) the mindreading capacity is cross-culturally universal.

The first step in my rebuttal was to note that, since Nagel aims to show that "neither ethnicity nor gender has a significant impact on knowledge ascriptions in general"(3), her contention in (ii) must be claiming that the mindreading capacity is cross-culturally universal in the strong sense. It is not enough that people in all cultures engage in mindreading; for her argument to work, they must do it in the same way. The second step was to note that "mindreading capacity" can be (and has been) construed in a variety of different ways. If it is construed broadly - including most of the abilities on Astington \& Baird's list - then it is singularly implausible that the mindreading capacity is cross-culturally universal, and patently false to claim, as Nagel does, that "mindreading capacities are generally thought to be cross-cultural universals."(17, emphasis added). So clearly, Nagel must have some narrower interpretation of "mindreading capacity" in mind. Unfortunately, she does not tell us what this interpretation is.

Since two of the three references Nagel offers in her defense of the claim that the mindreading capacity is cross-culturally universal are focused exclusively on the emergence of the ability to pass the false belief task, one might suppose that when Nagel talks about the "mindreading capacity" what she has in mind is the capacity to understand and attribute false beliefs, and whatever additional capacities come along with this. On that interpretation, there is indeed a growing consensus that (ii) is true. However, the view of many who share this consensus, including leading researchers like Callaghan et. al, Harris, Leslie and Wellman, is that what is universal is an early emerging "common starting point" or "beginning foundation" which is later supplemented with culturally specific principles. As a result, as Wellman notes in a passage quoted approvingly by Scholl and Leslie, adult "folk psychologies could be quite different from one another worldwide; each could be quite removed and advanced beyond, although grounded in, the initial framework assumptions of young children." (Scholl \& Leslie, 1999, 138)

In order for this consensus view to be of any help to Nagel, it must be the case that 
(iii) the psychological mechanisms responsible for attributions of knowledge are included in the "common starting point"

and that

(iv) subsequent cultural elaboration does not lead to significantly different patterns of knowledge attribution in different cultures.

But despite Nagel's loose talk about what is "generally thought," many leading researchers say little or nothing about (iii). Ian Apperly is a notable exception. On his account, there is a pancultural "original module" responsible for the attribution of "knowledge" which humans likely share with chimpanzees. However, on closer inspection, Apperly's view does not give Nagel what she needs. Apperly thinks that the concept of "knowledge" attributed by this original module does "not correspond fully with the adult human's understanding" of knowledge. Indeed, on Apperly's view, the concept of "knowledge" attributed by this original module is not even a propositional attitude! Moreover, even if Apperly's original module attributes knowledge (without scare quotes), Apperly's view gives us no reason to think that (iv) is true. Quite the opposite is the case; his view strongly suggests that (iv) is false.

I conclude that Nagel's attempt to defend (i) and (ii) is a failure. And since (i) and (ii) are crucial premises in her theoretical argument, her theoretical argument fails as well. According to Nagel, "neither ethnicity nor gender has a significant impact on knowledge ascriptions in general." But since her theoretical argument is the only support she offers for this sweeping conclusion, ${ }^{17}$ she has offered us no good reason to think this conclusion is true.

\section{Nagel's Strategy for Dealing with Data}

In the section of her paper in which Nagel develops her theoretical argument, she also discusses the Weinberg, Nichols and Stich (2001; hereafter WNS) paper which presented data supporting the claim that epistemic intuitions vary across ethnic groups, and she offers a brief overview of an unpublished experiment in which she and her collaborators failed to find any significant correlations between ethnicity or gender and knowledge ascriptions in most (but apparently not all) of the epistemological scenarios they employed. As I noted in the Introduction, I think it would be inappropriate to comment on Nagel's experiment at this time, since the details provided in her papers are very sketchy indeed. However, before closing, I do want to comment briefly on Nagel's strategy for dealing with data.

In critiquing the WNS experiment, Nagel offers a number reasons why the results reported might not actually indicate "underlying differences in intuitive competence."(19) "It is possible," she tells us, "that the 24 South Asian participants in the study were on average simply less engaged with these particular problems." Perhaps they were less likely to be philosophy majors, "and if participants were thinking of the series of puzzles as an exercise in applying

\footnotetext{
${ }^{17}$ Nagel's unpublished experimental work, even if entirely unproblematic, could not possibly support this sweeping conclusion about "knowledge ascriptions in general," since, like all experimental work in this area, it focuses on a relatively small number of examples and uses participants from a relatively small number of ethnic groups.
} 
philosophical methods, then the prospect of pondering these cases may have been less attractive to them."(19) Or perhaps the WNS Gettier scenario "resonated more with members of some ethnic groups than others."(19) Or perhaps "some groups found it easier to read." The first thing to say about all of this is that Nagel is surely right on every single point. Each of the possibilities she imagines could indeed be the case. And if they were, they would be good reasons to be skeptical about whether the WNS data are really telling us anything interesting about the participants' epistemic intuitions. The second thing to say is that, apart from noting that Asian students are much more likely than Whites to major in biology or engineering, Nagel gives us no reason to think that any of these possibilities are actually the case. The third thing to say is that this game is very easy to play. As I noted in section 2 , in just about every social science experiment there are lots of possible confounds that have not been ruled out.

Possibilities that, if actual, would prevent drawing any interesting conclusions from the data are, near enough, unlimited, while feasible controls, in any one experiment, are very limited indeed. So I predict that when we have a more complete account of Nagel's own experiment, it will be easy propose a long list of possibilities which, if they actually obtained, would provide good reason to be skeptical about whether her data are really telling us anything interesting about the participants' intuition.

As it happens, we don't have to wait until we have a more complete account of her experiment, since Nagel has already begun the process. Some of the data in her experiment is clearly to her liking, since it suggests that there are no ethnic or gender differences in responses to some of her scenarios. But some of her data is not to her liking, and when it isn't, once again, she assembles lists of possibilities that, if they obtained, would be reasons to discount the data. So, for example, almost $33 \%$ of her participants attributed knowledge on her "eight core Gettier cases" and almost 16\% ascribed knowledge in justified false belief cases. This looks like bad news for someone who maintains that "amateur judgments of ordinary knowledge, Gettier, skeptical pressure and false belief cases align roughly with the judgments of professionals" (27) since "if epistemologists have clear intuitions that Gettier cases involve a failure to know, then one might have expected a higher level of consensuality in amateur judgments of those cases." (21) But hey, don't worry. "Philosophers and others may have the same basic intuitive capacity to register the presence or absence of knowledge, but philosophers may be more motivated to read epistemic vignettes with an eye to exercising this capacity."(21-2) Is this possible? Of course it is. And it is also possible that "amateur participants who do not have the motivation of testing an epistemological theory may be less inclined to read closely." (22) In another example, Nagel reports, in a footnote, that she and her colleagues used the WNS Gettier case and found (just as WNS had) that the percentage of South Asians who attribute knowledge is substantially higher than the percentage of Whites who attribute knowledge. Is this apparent replication of one of the WNS findings problematic for someone who claims there are no ethnic differences in epistemic intuitions? Not at all. "Because we did not see any significant correlation between ethnicity and knowledge ascription across the eight other cases tested, it seems more reasonable to attribute the difference in response rates here to something like differing levels of engagement with this particular story...."(22) Is this possible? Yes, indeed. Did Nagel and colleagues make any effort to assess participants' levels of engagement? If so, she does not tell us. These are not the only examples. Between p. 19 and p. 26, Nagel uses the word 'may' ten times in conjuring possibilities that, if they obtained, would cast doubt on data that does not support her hypothesis, but at no point does she propose possibilities that, if they obtained, would cast doubt on data that appear to support her hypothesis. 
What is going on here? On an uncharitable interpretation, the rule that Nagel seems to be following in testing hypotheses empirically is:

If the data supports my view, all is well. If the data does not support my view, think up some possibility that, if it obtained would give us a reason to discount the data. No need to show that that possibility does obtain. The mere possibility that it obtains is enough to conclude that the data does not really pose a problem for my hypothesis.

But perhaps there is a more charitable interpretation of Nagel's selectivity in proposing possibilities that, if actual, would challenge empirical findings. If her theoretical argument were successful, we could predict, on the basis of that argument, that there should be little or no difference in epistemic intuition across individuals or groups. And if that prediction is true, then we would have good reason to go hunting for possible explanations of data suggesting that there are differences, but no reason to look for possibilities that would challenge data that show no differences. However, as I have argued in detail in Section 5, Nagel's theoretical argument is seriously mistaken. Thus it provides no justification at all for her practice of selectively challenging data that does not support her view. 


\section{$\underline{\text { References }}$}

Alexander, J. (forthcoming). Experimental Philosophy: An Introduction. Cambridge: WileyPolity.

Apperly, I. \& Butterfill (2009). Do humans have two systems to track beliefs and belief-like states? Psychological Review, 116, 4, 953-970.

Apperly, I. (2011). Mindreaders: The Cognitive Basis of "Theory of Mind”. Hove \& New York: Psychology Press.

Astington, J. \& Baird, J. (2005). Introduction: Why language matters. In J. Astington \& J.

Baird, eds., Why Language Matters for Theory of Mind. Oxford: Oxford University Press. 3-25.

Bauman, C. \& Skitka, L. (2006). Ethnic group differences in lay philosophies of behavior in the United States. Journal of Cross-Cultural Psychology37, 4, 438-445.

Buckwalter, W., \& Stich, S. (2011). Gender and the Philosophy Club. The Philosophers' Magazine (52), 60-65.

Callaghan, T., Rochat, P., Lillard, A., Claux, M., Odden, H., Itakura, S., Tapanya, S., \& Singh, S. (2005). Synchrony in the onset of mental-state reasoning. Psychological Science, 61, 5, 378-384

Cushman, F.A., L. Young \& J. Greene (2010). Multi-system moral psychology. In John Doris et al. (eds.), The Oxford Handbook of Moral Psychology. Oxford: Oxford University Press.

Duranti, Alessandro (2008). Further reflections on reading other minds. In Anthropology and the Opacity of Other Minds. Anthropological Quarterly, 81, 2, Spring 2008, pp. 483-494.

Feigenson, L., Dehane, S. \& Spelke, E. (2004). Core systems of number. Trends in Cognitive Sciences, 8,7, 307-314.

Fodor, J. (1998). Concepts: Where Cognitive Science Went Wrong, Oxford: Oxford University Press.

Gauvain, M. (1998). Culture, development, and theory of mind: Comment on Lillard (1998), Psychological Bulletin, 123, 1, 37-42.

Gilmore, C. \& Inglis, M. (2009). Automatic access to numerosity in symbolic arithmetic. Manuscript submitted for publication.

Goldman, A. (2007). Philosophical intuitions: Their target, their source, and their epistemic status. Grazer Philosophische Studien 74: 1-26.

Goldman, A. (2010). Philosophical naturalism and intuitional methodology (Romanell Lecture), Proceedings and Addresses of the American Philosophical Association, pp. 115-150. 
Hare, B., Call, J., Agnetta, B. \& Tomasello, M. (2000). Chimpanzees know what conspecifics do and do not see. Animal Behavior, 59, 771-785.

Hare, B., Call, J. \& Tomasello, M. (2001). Do chimpanzees know what conspecifics know and do not know? Animal Behavior, 61, 139-151.

Harris, P. (1990). The child's theory of mind and its cultural context. In G. Butterworth \& P. Bryant (eds.), Causes of Development: Interdisciplinary Perspectives. Hillsdale, NJ: Erlbaum.

Heine, S. (2008). Cultural Psychology. New York: W. W. Norton.

Henrich J., Heine S. J., Norenzayan A. (2010). The weirdest people in the world? Behavioral \& Brain Sciences, 33, 61-135.

Jackson, F. (1998). From Metaphysics to Ethics: A Defense f Conceptual Analysis. Oxford: Clarendon Press.

Koriat, A. (1975). Phonetic symbolism and feeling of knowing. Memory \& Cognition, 3(5), 545-548.

Koriat, A. (1976). Another look at the relationship between phonetic symbolism and the feeling of knowing. Memory \& Cognition, 4(3), 244-248.

Koriat, A. (1995). Dissociating knowing and the feeling of knowing: Further evidence for the accessibility model. Journal of Experimental Psychology: General, 124(3), 311-333.

Koriat, A. (2008). Subjective confidence in one's answers: The consensuality principle. Journal of Experimental Psychology: Learning, Memory, and Cognition, 34(4), 945-959.

Koriat, A. (2011). Subjective Confidence in Perceptual Judgments: A Test of the Self-Consistency Model. Journal of Experimental Psychology: General, 140(1), 117-139.

Lillard, A. (1997). "Other Folks' Theories of Mind and Behavior," Psychological Science, 8,4, 268-274.

Lillard, A. (1998). Ethnopsychologies: cultural variation in theories of mind, Psychological Bulletin, 123, 1, 3-32.

Lillard, A. (1999). "Developing a Cultural Theory of Mind: The CIAO Approach," Current Directions in Psychological Science, 8,2, 57-61.

Machery, E. (2008). The folk concept of intentional action: Philosophical and experimental issues. Mind and Language, 23, 2, 165-189.

Mercier, H., \& Sperber, D. (2009). Intuitive and reflective inferences. In J. Evans \& K. Frankish (eds.), In two minds: Dual processes and beyond. Oxford: Oxford University Press. 
Nado, J. (2011). Intuition and Inquiry. Ph.D. dissertation, Department of Philosophy, Rutgers University.

Nagel, J. (2011). Intuitions and experiments. Forthcoming in Philosophy and Phenomenological Research. Available at: http://philpapers.org/archive/NAGIAE.1.pdf . In this paper, I use the version posted at that URL and dated April 7, 2011.

Nagel, J. (forthcoming). Mindreading in Gettier cases and skeptical pressure cases. To appear in J. Brown \& M. Gerken (eds.), Knowledge Ascriptions. Oxford University Press.

Nelson, Katherine (2005). Language pathways into the community of minds. In J. Astington \& J. Baird, eds., Why Language Matters for Theory of Mind. Oxford: Oxford University Press. 2649.

Paul, R. (1995). Act and intention in Sherpa culture and society. In L. Rosen, ed., Other Intentions: Cultural Contexts and the Attribution of Inner States, Santa Fe, New Mexico: School of American Research Press, pp. 14-45.

Robbins, J. \& Rumsey, A. (2008). Introduction: Cultural and linguistic anthropology and the opacity of other minds. Anthropological Quarterly, 81, 2, 407-420.

Rosen, L. (1995). Introduction: The cultural analysis of others' inner states. In L. Rosen, ed., Other Intentions: Cultural Contexts and the Attribution of Inner States, Santa Fe, New Mexico: School of American Research Press, 3-11.

Scholl, B. \& Leslie, A. (1999). Modularity, development and 'theory of mind'. Mind and Language, 14, 1, 131-153.

Segall, M., Campbell, D.. \& Herskovits, M. (1966) The influence of culture on visual perception. Bobbs-Merrill.

Sinnott-Armstrong, W. \& Wheatley, T. (under review). Moral judgments are not unified: Why moral psychology needs a taxonomic approach.

Sloman, S. (1996). The empirical case for two systems of reasoning. Psychological Bulletin, 119(1), 3-22.

Sosa, E. (2009). A defense of the use of intuitions in philosophy. In D. Murphy \& M. Bishop, eds., Stich and His Critics. Oxford: Wiley-Blackwell.

Sosa, E (2010). Intuitions and meaning divergence. Philosophical Psychology, 23, 4, 419-426

Spelke, E, \& Kinzler, K. (2007). Core knowledge. Developmental Science, 10,1, 89-96.

Swain, S., Alexander, J., \& Weinberg, J. M. (2008). The instability of philosophical intuitions: Running hot and cold on Truetemp. Philosophy and Phenomenological Research, 76(1), 138-155. 
Vinden, P. (1999). Children's understanding of mind and emotion: A multi-culture study. Cognition \& Emotion, 13, 1, 19-48.

Weinberg, J. (2007). How to challenge intuitions empirically without risking skepticism. Midwest Studies in Philosophy, 31, 318-343.

Weinberg, J. S., Nichols, S., \& Stich, S. (2001). Normativity and Epistemic Intuitions. Philosophical Topics, 29 (1), 429-460.

Wellman, H. (1998). Culture, variation, and levels of analysis in folk psychologies: Comment on Lillard (1998). Psychological Bulletin, 123, 1, 33-36. 

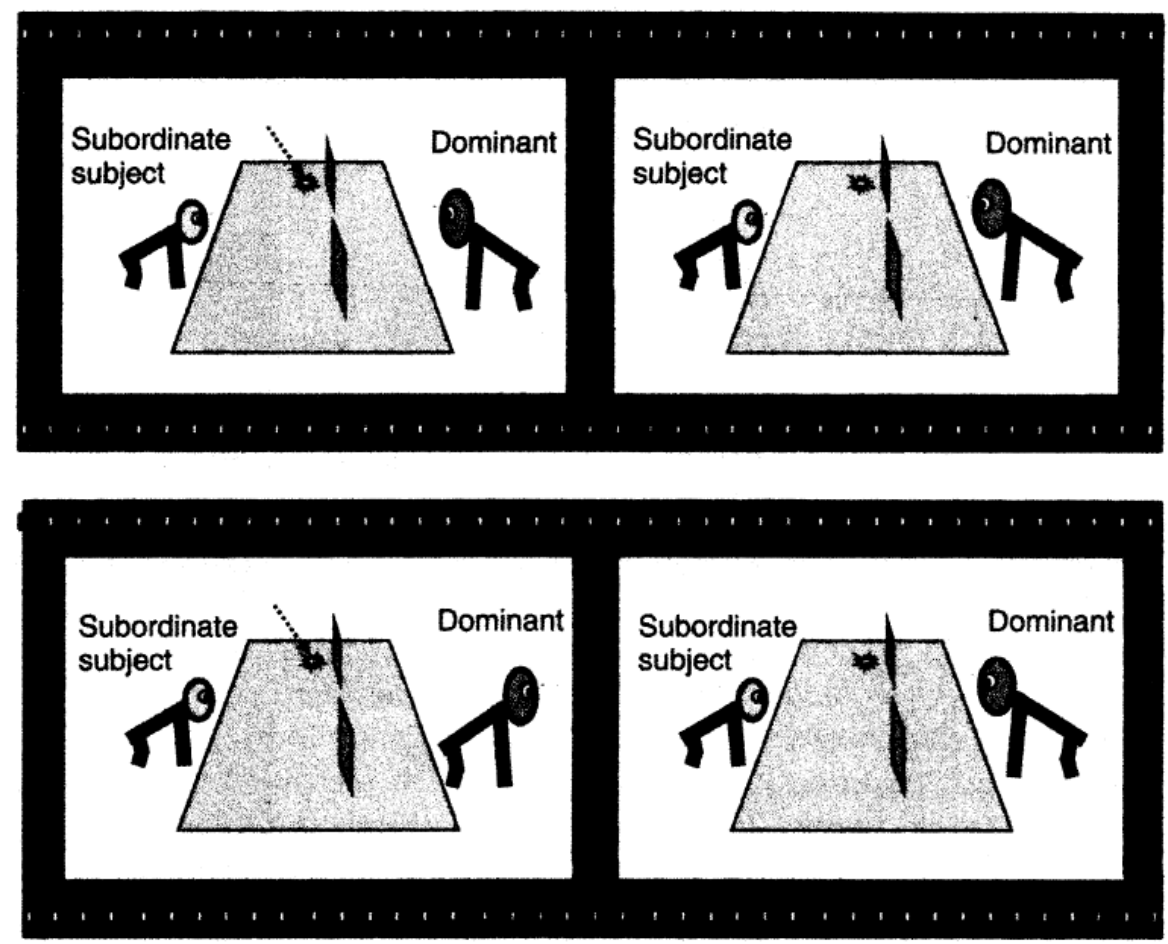

Figure 1: Hare, Call \& Tomasello's (2001) test of whether chimpanzees track what others "know". Subordinate subjects were less likely to approach the food when the dominant chimpanzee saw where it was hidden (upper two panels) compares with when the dominant did not see (lower two panels). [From Apperly (2011), p. 49] 